\title{
Multiple sclerosis flare secondary to COVID-19 vaccine, a case report
}

\author{
ahmad matarneh ${ }^{1}$, Alia Al Battah², Riyadh Hammamy², and Mohammed alhatou ${ }^{2}$ \\ ${ }^{1}$ Hamad medical corporation \\ ${ }^{2}$ Hamad Medical Corporation
}

July 25, 2021

\begin{abstract}
We report a 60-year-old lady known to have a stable MS disease. Who presented with right-hand weakness on the same day of COVID 19 vaccine administration. MRI showed a relapse, and she was treated with steroids with improvement in her symptoms. neurological side effects can occur following COVID 19 vaccines
\end{abstract}

Multiple sclerosis flare secondary to COVID-19 vaccine, a case report

Alia Hani Al-Battah 1, Ahmad S matarneh 1, Riyadh Hammamy 1, Mohammed Alhatou 2 1. Internal Medicine department, Hamad medical corporation, Doha, Qatar.

2. Department of neurology, Hamad medical corporation, Doha, Qatar.

*Corresponding Author

Dr. Alia Hani Mohammed Al-Battah

Department Internal Medicine.

Hamad Medical Corporation.

Al Rayyan street.

Doha, Qatar.

Tel: +97450441190

Fax: -44397857

E-mail: aalbattah3@hamad.qa

Keywords:

Multiple sclerosis, COVID 19 vaccine, Pandemic, neurology

Key Clinical message:

COVID 19 Vaccines have a good safety and immunogenicity profiles. However, they can result in different side effects. One of the neurological side effects is multiple sclerosis relapse. However, its exact incidence is not currently known.

\section{Abstract: \\ Introduction:}


COVID 19 is a newly emerging disease. It has affected millions of people and resulted in significant morbidity and mortality rates. As a result, efforts have been made to find a cure. In December 2020, the first COVID vaccines were made with impressive efficacy but an unclear safety profile as further studies are needed to elaborate and uncover all potential side effects.

\section{Case presentation:}

We report a 60-year-old lady who is known to have a stable MS disease. Who presented with right hand decreased strength during the same day of COVID vaccine administration. MRI revealed features consistent with relapse, and she was treated with steroids with improvement in her symptoms.

\section{Conclusion:}

COVID 19 Vaccines are newly introduced. They have good safety and immunogenicity profiles. However, they can result in different side effects, as is the case with all other vaccines. Neurological side effects can occur following COVID-19 vaccines. However, their frequency is not well studied yet. One of those side effects is MS relapse. However, its exact incidence is not currently known.

\section{Introduction:}

COVID-19 has newly emerged from Wuhan, China, in December 2019. It became a pandemic after infecting millions of people causing significant morbidity and mortality rates in the world. It caused so many challenges on the social, psychological, and financial aspects. (1) Moreover, it made changes in the way healthcare is delivered. It is primarily a disease of the respiratory tract that causes a wide variety of presentations, ranging from asymptomatic to a more severe picture with ARDS and even death. (2) It is worth mentioning that it can infect other systems as described in the literature. Research has been going on ever since starting to find new treatments or approaches to help cure it. In December 2020, the first covid vaccines were introduced after extensive research. (3) They utilized a new technology which is the mRNA-based vaccines. They have been effective with a protection rate as high as $95 \%$ and an impressive safety profile. (4) However, they have been found to cause many side effects, ranging from simple myalgias, headaches, fever, pain at the injections site. To more severe side effects such as the development of SARS and ARDS. (5) It was also found that they have multi-system side effects. CNS-related side effects were described in literature and trials. It can cause encephalitis, demyelination. And further studies are needed to unveil all the potential side effects. (6) We report a 61-year-old lady who previously had stable disease and then developed MS flare the same day she received the COVID vaccines.

\section{Case presentation:}

A 60-year-old lady, known to have multiple sclerosis, presented with right-hand weakness a few hours after receiving the 1st dose of COVID-19 vaccine (Pfizer)

Her hand weakness was progressive; she was unable to hold or pick up objects.

She had no other neurological deficits, no numbness or other limb weakness, no visual loss or eye pain, No bladder or bowel dysfunction.

She was diagnosed with multiple sclerosis in 1996, was on interferon, but stopped in 2015.

She was in remission for the past eight years without clinical deterioration or new MRI changes.

On examination, the patient had normal vital signs

The neurological exam was remarkable for decreased handgrip and thumb opposition strength. There was no muscle wasting or reduced sensation. The rest of the neurological examination, cardiovascular, pulmonary, and abdominal examinations were normal.

Labs showed leukopenia and mildly elevated CRP, otherwise normal Hgb, platelets, urea, creatinine, and electrolytes (Table 1). 
CT head was done, which showed Ill-defined focal hypodense lesions in the bilateral parietal white matter, likely suggesting chronic demyelinating lesions.

MRI head and MRI spinal cord with contrast were done, which showed old demyelinating lesions with a newly described lesion measuring approximately $10 \times 9 \mathrm{~mm}$ size with restricted diffusion) Figure 1 and 2, and enhancement on contrast study suggestive of activity figure 3 .

She received a 3-days course of intravenous methylprednisolone $500 \mathrm{mg} /$ day. Her power improved through her hospital stay and was discharged home.

Discussion:COVID 19 has resulted in a lot of morbidity and mortality all over the world. As a new disease entity, the evidence on the pathogeneses, potential complications, and treatment are growing daily. 7) COVID 19 vaccines were recently produced and given to the population after an emergency approval from the FDA. New technology has been introduced, which is mRNA-based vaccines. 8) They are associated with many variable side effects, ranging from simple ones to potentially more severe or life-threatening ones. They were described to cause myalgias, fever, general fatigue. But not uncommonly, they were seen to cause covid infection, with potential ARDS and death. 9) Vaccines, in general, have been linked to various side effects, such as the development of GBS and CNS Side effects. One other aspect that should be considered is that vaccines can cause several side effects for patients with autoimmune conditions. For example, the flu vaccine has been linked before to causing a flare of multiple sclerosis. (10) COVID 19 vaccines are continuously being studied, and more side effects will be identified as more and more people receive the Vaccine. (11) They have been found to cause several CNS side effects, such as weakness, numbness, ataxia, and more drastic presentations such as encephalomyelitis and acute demyelination syndrome with GBS. Moreover, it was described in recently published studies that MS flare could be associated with COVID vaccines. The frequency is not precise yet, and more and more studies are needed. (12) Additionally, there is some evidence to suggest a temporal relationship between MS flare and the receipt of various vaccines, including those for rabies, hepatitis (Hep) A and B, polio, and influenza [13]. Such cases are infrequent, and the establishment of causality in most of these cases has proved controversial.

Multiple sclerosis is an autoimmune disease with unclear etiology. The diagnosis is usually made as a combination of clinical and radiological findings. Treatment is done with pulse steroids; high-dose IV steroids. (14) The risk of relapses usually decreases with age and people who had stable disease (include more on the relapse). Moreover, it is known that MS flares decrease with age, and it is unlikely in patients who have had stable diseases for many years. (15)

Our patient was diagnosed with MS more than 20 years ago and had stable disease with no relapses. The most recent relapse was 18 years ago, and she was kept off medications. Unfortunately, on the same day that she received the COVID vaccine, she had a recurrence of symptoms. MRI Was done, and it revealed an active lesion in the left periventricular region. Her symptoms improved significantly after she started pulse steroids with a resolution of her symptoms upon follow-up.

\section{Conclusion:}

COVID 19 pandemic has resulted in many burdens on the social, financial, psychological, and physical aspects. COVID 19 vaccines were recently introduced to help combat the effects of this pandemic. These vaccines are effective with great immunogenicity; however, their complete safety is not yet established. Therefore, the potential benefits of the vaccines are outweighed by the potential benefits, and more studies are needed to further unveil all the potential risks from these vaccines. MS risk of Relapse decreases with age and those with stable disease. However, the development of MS relapse following the vaccine administration should be considered a potential side effect.

Data availability statement:The data that support the findings of this study are available from the corresponding author upon reasonable request.

Conflict of interest: The authors have no conflict of interest to declare. 


\section{Funding information}

Qatar national library - Qatar foundation

\section{Ethical Approval}

The case report was approved by Hamad medical corporation, MRC number MRC-04-21-387.

\section{Acknowledgments}

We thank the internal medicine department at Hamad Medical Corporation for giving us the opportunity and support to conduct this work.

\section{Authors Contribution:}

Alia Hani Al Battah: History and physical examination, literature review, manuscript writing Ahmad S Matarneh: History and physical examination, literature review, manuscript writing Riyadh Hammamy : Clinical Care Mohammed Alhatou: clinical care, manuscript writing, literature review and mentor

References

Lu H, Stratton CW, Tang YW. Outbreak of pneumonia of unknown etiology in Wuhan, China: The mystery and the miracle. Journal of medical virology. 2020 Apr;92(4):401.

Esakandari H, Nabi-Afjadi M, Fakkari-Afjadi J, Farahmandian N, Miresmaeili SM, Bahreini E. A comprehensive review of COVID-19 characteristics. Biological procedures online. 2020 Dec;22:1-0.

Cascella M, Rajnik M, Aleem A, Dulebohn S, Di Napoli R. Features, evaluation, and treatment of coronavirus (COVID-19). StatPearls. 2021 Apr 20.

Kaur SP, Gupta V. COVID-19 Vaccine: A comprehensive status report. Virus research. 2020 Aug 13:198114.

Kim JH, Marks F, Clemens JD. Looking beyond COVID-19 vaccine phase 3 trials. Nature medicine. 2021 Feb;27(2):205-11.

Lu L, Xiong W, Mu J, Zhang Q, Zhang H, Zou L, Li W, He L, Sander JW, Zhou D. Neurological side effects of COVID-19 vaccines are rare. Acta Neurologica Scandinavica. 2021 Jul;144(1):111.

Lotfi M, Hamblin MR, Rezaei N. COVID-19: Transmission, prevention, and potential therapeutic opportunities. Clinica chimica acta. 2020 Sep 1;508:254-66.

Shahcheraghi SH, Ayatollahi J, Aljabali AA, Shastri MD, Shukla SD, Chellappan DK, Jha NK, Anand K, Katari NK, Mehta M, Satija S. An overview of vaccine development for COVID-19. Therapeutic delivery. 2021 Mar;12(3):235-44.

Anand P, Stahel VP. Review the safety of Covid-19 mRNA vaccines: a review. Patient safety in surgery. 2021 Dec;15(1):1-9.

Huynh W, Cordato DJ, Kehdi E, Masters LT, Dedousis C. Post-vaccination encephalomyelitis: literature review and illustrative case. Journal of Clinical Neuroscience. 2008 Dec 1;15(12):1315-22.

Kadali RA, Janagama R, Peruru S, Malayala SV. Side effects of BNT162b2 mRNA COVID-19 vaccine: A randomized, cross-sectional study with detailed self-reported symptoms from healthcare workers. International Journal of Infectious Diseases. 2021 May 1;106:376-81.

Goss AL, Samudralwar RD, Das RR, Nath A. ANA investigates: neurological complications of COVID-19 vaccines. Annals of neurology. 2021 May;89(5):856-7.

Confavreux C, Suissa S, Saddier P, Bourdès V, Vukusic S. Vaccinations and the risk of relapse in multiple sclerosis. New England journal of medicine. 2001 Feb 1;344(5):319-26.

Dobson R, Giovannoni G. Multiple sclerosis-a review. European journal of neurology. 2019 Jan;26(1):27-40. 
Goldenberg MM. Multiple sclerosis review. Pharmacy and Therapeutics. 2012 Mar;37(3):175.

\section{Hosted file}

figures.docx available at https://authorea.com/users/418217/articles/531564-multiplesclerosis-flare-secondary-to-covid-19-vaccine-a-case-report 